\title{
Lactobacillus rhamnosus GG
}

National Cancer Institute

\section{Source}

National Cancer Institute. Lactobacillus rhamnosus GG. NCI Thesaurus. Code C61097.

A live specific strain of the species, Lactobacillus rhamnosus (a subspecies of

Lactobacillus casei) with probiotic activity. When administered orally, Lactobacillus

rhamnosus GG adheres to the mucous membrane of the intestine and may help to restore the balance of the GI microflora; promote gut-barrier functions; diminish the production of carcinogenic compounds by other intestinal bacteria; and activate the innate immune 